\title{
Freeze-Dried Therapeutic Microbubbles: Stability and Gas Exchange
}

Radwa H. Abou-Saleh, Aileen Delaney, Nicola Ingram, Damien V. B. Batchelor, Benjamin R. G. Johnson, Antonia Charalambous, Richard J. Bushby, Sally A. Peyman, P. Louise Coletta, Alexander F. Markham, and Stephen D. Evans*

Cite This: ACS Appl. Bio Mater. 2020, 3, 7840-7848

Read Online

ACCESS | 山ll Metrics \& More 回国 Article Recommendations

Supporting Information

ABSTRACT: Microbubbles (MBs) are widely used as contrast enhancement agents for ultrasound imaging and have the potential to enhance therapeutic delivery to diseases such as cancer. Yet, they are only stable in solution for a few hours to days after production, which limits their potential application. Freeze-drying provides long-term storage, ease of transport, and consistency in structure and composition, thereby facilitating their use in clinical settings. Therapeutic microbubbles (thMBs) consisting of MBs with attached therapeutic payload potentially face even greater issues for production, stability, and well-defined drug delivery. The ability to freeze-dry thMBs represents an important step for their translation to the clinic. Here, we show that it is possible to freeze-dry and reconstitute thMBs that consist of lipidcoated MBs with an attached liposomal payload. The thMBs were produced microfluidically, and the liposomes contained either calcein, as a model drug,

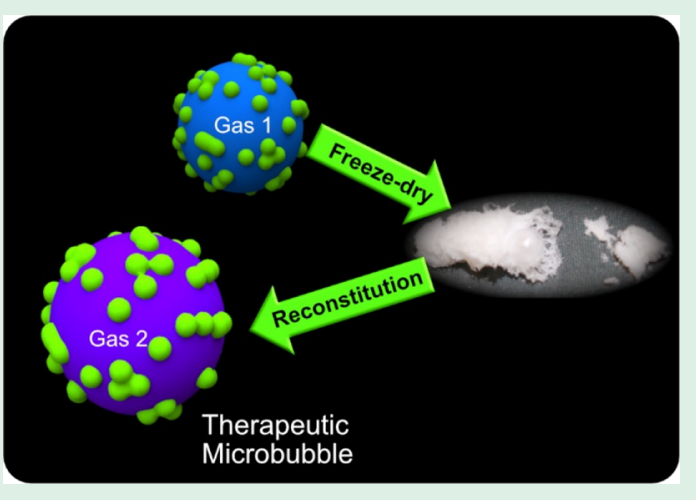
or gemcitabine. The results show that drug-loaded thMBs can be freeze-dried and stored for at least 6 months. Upon reconstitution, they maintain their structural integrity and drug loading. Furthermore, we show that their in vivo echogenicity is maintained post-freeze-drying. Depending on the gas used in the original bubbles, we also demonstrate that the approach provides a method to exchange the gas core to allow the formulation of thMBs with different gases for combination therapies or improved drug efficacy. Importantly, this work provides an important route for the facile off-site production of thMBs that can be reformulated at the point of care.

KEYWORDS: microbubbles, drug delivery, sucrose, freeze-drying, stability, liposomes, microfluidics

\section{INTRODUCTION}

Microbubbles (MBs) are widely used as contrast enhancement agents in ultrasound (US) imaging and typically consist of a perfluorocarbon gas core and a biocompatible shell (e.g., protein, phospholipid, or polymer). ${ }^{1-4}$ The enhanced US contrast arises from the high mismatch in acoustic impedance between the gas core and the surrounding medium. The properties of the shell and the encapsulated gas affect the MB stability in vitro and in vivo as well as their US response. ${ }^{5-10}$ MBs undergo US-induced oscillations that result in microstreaming, or at higher amplitude, MB destruction. ${ }^{11,12}$ These processes can create pores in cell membranes and enhance the passage of drug molecules across the cell membrane. These characteristics mean that drugs co-delivered with MBs and exposed to focused US can promote localized and enhanced therapeutic delivery. ${ }^{2,13,14}$ Furthermore, it has been demonstrated that it is possible to attach drug-filled liposomes, oil nanodroplets, genes, or lipid conjugated drugs to the shell of $\mathrm{MBs},{ }^{15-19}$ leading to the formulation of therapeutic microbubbles (thMBs). The thMB complex can be further targeted toward the required delivery site using antibodies, or other targeting ligands, for localized delivery. ${ }^{20}$
Typically, for safe use in vivo, MBs should have a size no more than $10 \mu \mathrm{m}$ in diameter. There are numerous preparation protocols for MBs that provide control over the size distributions that can be achieved. ${ }^{21,22}$ Sonication and mechanical agitation are the conventional methods for producing $\mathrm{MBs}$ at a high concentration of $\sim 10^{9}-10^{10} \mathrm{MB} /$ $\mathrm{mL}$ but have poor control over the $\mathrm{MB}$ size. ${ }^{2,24}$ A flowfocused microfluidic (MF) technology has been successful for the production of monodisperse $\mathrm{MBs}$ with size control in a range of $2-50 \mu \mathrm{m}$. However, these $\mathrm{MBs}$ are susceptible to changes in size off-chip and are produced at relatively low concentrations $\left(\sim 10^{6}-10^{8} \mathrm{MB} / \mathrm{mL}\right) .^{25-28}$ We have previously developed a rapid pressure drop on-chip method, which led to the production of $\mathrm{MB}$ populations with a size distribution in the range $0.5-3 \mu \mathrm{m}$ as well as a "one-pot" MF production of

Received: August 11, 2020

Accepted: October 15, 2020

Published: October 30, 2020 


\section{Scheme 1. Process of Freeze-Drying Microspray thMBs ${ }^{a}$}

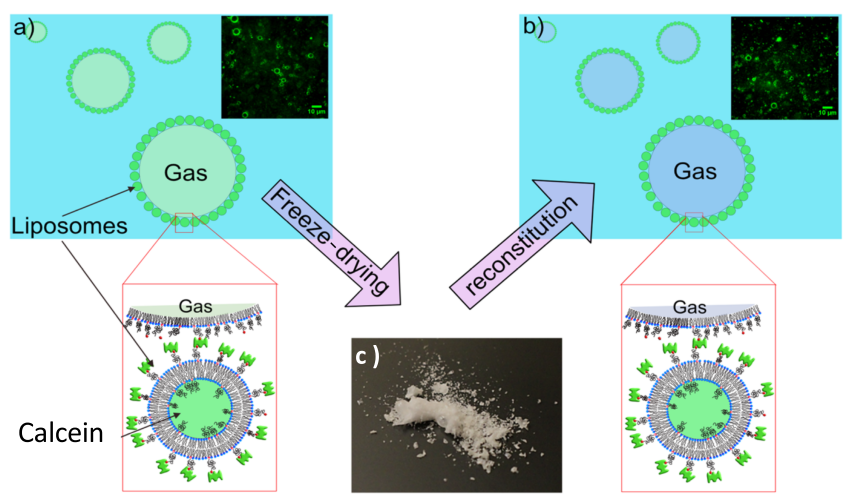

${ }^{a}$ The thMBs are freeze-dried as shown schematically in (a) and can be stored until required. (b) The thMBs can be reconstituted with the same gas core or with a different one as required. The image in (c) shows the freeze-dried thMB powder.

liposome-loaded $\mathrm{MBs}$ at high concentrations $\left(\sim 10^{9} \mathrm{MB} /\right.$ $\mathrm{mL})$.

The in vitro and in vivo stability of MBs has been extensively studied by different groups, and the role of the gas type, lipid shell composition, and saturation have all been shown to be important for controlling MB lifetime. ${ }^{6,9,30-33}$ Although MBs can often be stable for many hours at $4{ }^{\circ} \mathrm{C}$, this is still relatively short for therapeutic agents and generally means that treatment protocols would require $\mathrm{MB}$ production directly before use, at the point of care. If thMBs could be freeze-dried (FD) at production and subsequently reconstituted at the point of use, this would offer advantages of better control of drug/MB concentration and simpler protocols for administration and would also allow off-site production at lower cost.

FD has been widely used for increasing shelf life, preserving sensitive samples such as vaccines and proteins, stabilizing pharmaceutical products such as liposomes, and maintaining biological activities after reconstitution. ${ }^{34-38}$ FD has been used as a method for drug loading preformulated microparticles, ensuring uniform and high drug loading. ${ }^{39}$ Although the FD process is relatively simple, ensuring that the reconstituted product is the same form as the starting one is critical. To this end, sugars are frequently used as cryoprotectants. They work by forming a glassy layer around individual particles, preventing interaction between them and creating a protective barrier from the mechanical stresses exerted by the formation of ice crystals. ${ }^{40}$ Glucose, sucrose, trehalose, and mannitol have been widely used. ${ }^{38}$ It has been shown that sucrose and lactose are better protectants for systems involving lipid layers, where they form a glassy layer near the polar head groups, on the inside and outside of the liposomes, that protects the lipid bilayer from collapse under the stress caused by the FD process. $^{41}$

A vitrification theory is the leading explanation for how cryoprotectants work, and as explained by Franks, ${ }^{36}$ freezing and drying should occur below the glass transition temperature $T_{\mathrm{g}}$ to avoid the collapse of the structure. Freezing is the first step in the process where most of the water is removed from the solution as ice crystals begin to form. The methods of freezing vary, either by using liquid nitrogen to flash freeze the sample $^{42}$ or by cooling the sample within an FD machine at a steady cooling profile of $1{ }^{\circ} \mathrm{C} / \mathrm{min} .{ }^{36}$ As the water is removed, the concentration of the cryoprotectant increases and the solution becomes increasingly viscous. This is then followed by drying under vacuum at a temperature below $T_{\mathrm{g}}$, where the sublimation of ice from the sample takes place.

Freeze-drying has been used for the preparation of MBs for contrast-enhanced US imaging. SonoVue, a clinically used contrast agent produced by Bracco, uses freeze-drying or spraydrying of lipids, which are subsequently mechanically agitated in the presence of a low solubility gas and aqueous carrier to create MBs with a size of $0.5-10 \mu \mathrm{m}$ just before use. ${ }^{43}$ Sonozoid, produced by GE Healthcare, uses sucrose during the lyophilization of lipid-coated perfluorobutane microspheres, which are reconstituted by manual mixing. ${ }^{44,45}$ The method was patented by Borden in 2010 for freeze-drying in-houseproduced MBs as a method for possible gas-core exchange. ${ }^{46}$ The freeze-drying concept has also been applied to MBs in the presence of cryoprotectants such as sucrose, where the bubbles were found to form cavities in the cryoprotectant matrix. After refilling the vial with the appropriate gas, these cavities are filled and MBs are released upon rehydration. This has been used by Wheatley's group in surfactant-based contrast agents (such as SE61, composed of Span60 and water-soluble vitamin E) to change the octa-fluoropropane gas core to $\mathrm{O}_{2}$ to increase the $\mathrm{O}_{2}$ level in hypoxic tissue upon delivery ${ }^{47}$ and, by the same group, to preserve the integrity of contrast agents with the nonionic ST68. ${ }^{42}$ The freeze-drying process has also been employed to increase the shelf life of polymeric MBs. In this study, they demonstrated that sucrose was better than glucose and other cryoprotectants for rhodamine-B dye loading into the polymeric MBs with all of the dye being retained after lyophilization with no leakage in the aqueous suspension. ${ }^{48}$

In 2019, Unga et al. freeze-dried octa-fluoropropane lipidbased $\mathrm{MBs}$ and claimed that a modified composition of DSPC:DSPG:DSPE-PEG 2000 formulated MBs with sucrose was a versatile formulation for improved stability of MBs after freeze-drying. ${ }^{49}$ They also examined scaling up the production process in-line with potential clinical applications and saw no changes in composition or toxicity of the MBs after 1 year of storage. $^{50}$ However, to date, the freeze-drying of drug-loaded thMBs has not been reported and the potential for loss of liposomes from the $\mathrm{MB}$ shell and/or leakage of liposomal content must be considered.

Here, we investigate the feasibility of freeze-drying and reconstituting thMBs, i.e., MBs loaded with liposomal payloads (Scheme 1). We evaluate the quality of MBs reconstituted up to 6 months post-FD and test MB stability and lifetime both in vitro and in vivo. The FD process not only provides a long shelf 
life and facilitates easy shipping of thMB products but also provides the ability to control the MB concentration of the reconstituted population and the possibility to exchange the encapsulated gas after the FD process. This is considered a vital step toward translation of thMBs from the lab toward clinical trials and commercialization.

\section{MATERIALS AND METHODS}

2.1. Materials. The lipids used for $M B$ production, 1,2dipalmitoyl-sn-glycero-3-phosphocholine (DPPC), 1,2-distearoyl-snglycero-3-phosphoethanolamine- $N$-biotin-[methoxy(polyethylene-glycol)-2000] (DSPE-B-PEG 2000 ), 1,2-distearoyl-sn-glycero-3-phosphocholine (DSPC), and cholesterol, were purchased from Avanti Polar Lipids (Alabaster, AL, USA). Texas Red 1,2-dihexadecanoyl-snglycero-3-phosphoethanolamine and triethylammonium salt (DHPETexas red) were purchased from Invitrogen Life Technologies Ltd. (Paisley, UK). Stock solutions of lipids were prepared by dissolving the powder lipids as received in 50/50 chloroform/methanol. The biotin-binding protein NeutrAvidin was purchased from Life Technologies (Paisley, UK). Tetradecafluorohexane $\left(\mathrm{C}_{6} \mathrm{~F}_{14}, 99 \%\right.$ purity), cobalt(II) chloride $\left(\mathrm{CoCl}_{2},>98 \%\right.$ purity), gemcitabine hydrochloride ( $>98 \%$ purity), $\mathrm{NaCl}$, sucrose, and glucose were purchased from Sigma-Aldrich (St. Louis, MO, USA). Calcein and Triton X-100 were purchased from Fisher Scientific (Fisher Scientific UK Ltd., UK).

2.2. Microbubble Production and Characterization. A Horizon instrument (Horizon v3, Leeds, UK) ${ }^{29}$ was used for all MB production with a polymethylmethacrylate (PMMA) flowfocused MF chip designed in-house and fabricated by Epigem (Redcar, UK) to prepare MBs according to methodologies previously published. 29,51 Both the liquid flow rate and gas (perfluorobutane $\left(\mathrm{C}_{4} \mathrm{~F}_{10}\right)$ from $\mathrm{F} 2$ chemicals (Preston, $\left.\mathrm{UK}\right)$ ) pressure were controlled by a p-pump, a pressure-based pumping system from Dolomite (Royston, UK).

Liposomes were produced with different encapsulations, such as calcein as a fluorescent model drug as well as chemotherapy drugs such as gemcitabine (gem.) and SN38. These were manufactured by extruding a phospholipid solution through a polycarbonate membrane using an Avanti mini extruder (Alabaster, AL, USA). ${ }^{12,15}$ Briefly, a mixture of DSPC, cholesterol, and DSPE-B-PEG 2000 was dissolved together and mixed in the ratio $60: 35: 5 \mathrm{~mol} \%$, respectively. Chloroform was evaporated under vacuum for $\sim 24 \mathrm{~h}$, after which the lipids were re-suspended in a suitable buffer solution, depending on the encapsulant. The homogeneous solution was then extruded at $60{ }^{\circ} \mathrm{C}$ through a $400 \mathrm{~nm}$ membrane seven times and then through a $200 \mathrm{~nm}$ membrane 21 times. The liposomes were then cleaned either by centrifugation at $90 \mathrm{Kg}$ at $4{ }^{\circ} \mathrm{C}$ for $60 \mathrm{~min}$ in the case of calcein and gem. to remove un-encapsulated drug or by filtration through a 200 $\mathrm{nm}$ membrane in the case of SN38 liposomes.

Liposome size distribution and concentration were measured on a NanoSight NS300 (Malvern Panalytical, UK). The liposome sample was diluted 100,000 $\times$ in PBS, and the measurement was made at 25 ${ }^{\circ} \mathrm{C}$. For each sample, three $60 \mathrm{~s}$ videos were captured.

MBs were prepared as previously described. ${ }^{6}$ Briefly, DPPC and DSPE-B-PEG ${ }_{2000}$ were mixed together at $95: 5 \mathrm{~mol} \%$, respectively. After evaporation of the chloroform, the lipids were re-suspended in MilliQ water containing $1 \%$ glycerol, $10 \%$ sucrose, and $4 \mathrm{mg} / \mathrm{mL}$ $\mathrm{NaCl}$ in a $\sim 1 \mathrm{~mL}$ vial using an ultrasonic bath for about $1 \mathrm{~h}$. A total of $10 \mu \mathrm{L}$ of $\mathrm{C}_{6} \mathrm{~F}_{14}$ was added to the homogeneous solutions prior to use in the Horizon (MB maker). ${ }^{6}$

ThMBs were prepared using the "one-pot" production method previously described, ${ }^{12,26}$ in which $100 \mu \mathrm{L}$ of the premade liposomal solution containing $\sim 1 \times 10^{11}$ liposomes was incubated with $5 \mu \mathrm{L}$ of $42 \mathrm{M}$ stock NeutrAvidin for $20 \mathrm{~min}$. The liposomal mix was then incubated with the $\mathrm{MB}$ phospholipid solution containing the $10 \%$ sucrose used to prepare the $\mathrm{MBs}$ for further $20 \mathrm{~min}$, after which $\mathrm{C}_{6} \mathrm{~F}_{14}$ was added and thMBs were prepared with the Horizon platform.

After preparation of MBs or thMBs, the sample was flash frozen in liquid nitrogen for $20 \mathrm{~min}$. The samples were subsequently placed in a
mini-Lyotrap bench top freeze dryer (LTE Scientific Ltd., Oldham, $\mathrm{UK}$ ), which was pre-cooled to $-55{ }^{\circ} \mathrm{C}$ and was maintained under vacuum at $9 \times 10^{-3} \mathrm{mBar}$ for $48 \mathrm{~h}$. The process is shown schematically in Scheme 1. After freeze-drying, the vials were sealed under vacuum and stored at $4{ }^{\circ} \mathrm{C}$. The image in Scheme $1 \mathrm{c}$ shows the typical powdery appearance of the lyophilized thMB sample.

To re-suspend the freeze-dried sample, the head space of the vial was filled with $\mathrm{C}_{4} \mathrm{~F}_{10}$ gas for $3 \mathrm{~min}$ at $700 \mathrm{mbar}$ gas pressure. Typically, $1 \mathrm{~mL}$ of buffer was then added and the solution was gently shaken by hand until the dried cake of MBs was fully reconstituted (Scheme 1b).

For each MB population formed, a $10 \mu \mathrm{L}$ sample was diluted 10 fold to facilitate counting and sizing of the MBs. A total of $30 \mu \mathrm{L}$ of this $\mathrm{MB}$ solution was collected from the middle of the homogeneous sample and introduced in a $50 \mu \mathrm{m}$ height chamber on a glass slide. The MBs were allowed to rise for $\sim 1 \mathrm{~min}$ before images were acquired. An inverted microscope (Nikon, Japan) was used to image the $\mathrm{MBs}$ on a $60 \times$ magnification, and images were captured using a CCD camera (DS-Fil 5 Mpixel, Nikon, Japan). Twenty images for each sample were taken from random locations across the chamber, and the concentration and size distribution were obtained using a custom MATLAB (MathWorks, US) script utilizing the Image Analysis Toolbox..$^{52}$

2.3. Long-Term Stability of the Freeze-Dried MBs and In Vitro and In Vivo Stability of the Reconstitutes. For studies of the long-term stability of MBs, freeze-dried samples were stored in sealed vials at $4{ }^{\circ} \mathrm{C}$, and a sample was examined every month for 6 months. For each sample, MBs were reconstituted and their concentration and size distribution were determined.

To determine the time frame for using MBs after reconstitution, the in vitro stability of the reconstituted MBs was tested by counting $\mathrm{MB}$ number and size every $15 \mathrm{~min}$ from a vial of MBs kept at room temperature.

The in vivo stability/lifetime of the MBs and gem.-loaded thMBs was tested as previously described. ${ }^{6}$ CD1 nude male mice aged 7-9 weeks (Charles River Laboratories) were housed in individually ventilated cages with free access to food and water. All experiments were performed following local ethical approval and in accordance with the Home Office Animals (Scientific Procedures) Act 1986. Mice were anaesthetized using isofluorane, and a 27 -gauge tail vein catheter was inserted. The aorta and inferior vena cava were imaged using a RMV 704 transducer $(40 \mathrm{MHz})$ attached to a Vevo 770 highfrequency ultrasound machine (FUJIFILM VisualSonics). Pulse Wave Doppler imaging was used to confirm the pulsatile flow in the aorta. A syringe driver was used to inject $3 \times 10^{7} \mathrm{MBs}$ in $100 \mu \mathrm{L}$ volume at 0.6 $\mathrm{mL} /$ minute. Cine loops of the injection and for the next $15 \mathrm{~min}$ with $1 \mathrm{~min}$ gaps between each cine loop were recorded in contrast mode at $50 \%$ power.

2.4. Drug Leakage Assay. Calcein-loaded liposomes were used to test drug leakage from liposomes post-reconstitution. The calcein was encapsulated at $0.5 \mathrm{mM}$, a concentration at which it is fluorescent. $\mathrm{CoCl}_{2}$ was used as a quencher that does not cross the membrane but will quench fluorescence from any calcein that has leached into the bulk solution surrounding the liposomes. ${ }^{53}$

A Spectramax M2e microplate reader set with excitation/emission wavelengths of calcein $495 / 515 \mathrm{~nm}$ was used to measure the fluorescence intensity using a 96-well opaque plate. Three groups of MBs loaded with calcein liposomes were tested: one without any $\mathrm{CoCl}_{2}$ in the bulk solution to measure the intensity of encapsulated calcein, the second had $\mathrm{CoCl}_{2}$ and $50 \mu \mathrm{L}$ of ethanol to damage the liposome membrane and release the encapsulated calcein, and the third with $\mathrm{CoCl}_{2}$ only to quench any leaked calcein in the bulk solution over time. Samples were tested every $30 \mathrm{~min}$ for $3 \mathrm{~h}$. Each of these groups was analyzed in triplicate at each time point using samples before and after freeze-drying and repeated three times with three different samples.

2.5. Gas Exchange. $\mathrm{MBs}$ with $\mathrm{C}_{4} \mathrm{~F}_{10}$ or $\mathrm{SF}_{6}$ (BOC, UK) gas cores were prepared microfluidically and FD as described above. Post-FD, it was possible to reconstitute the MBs with different gaseous cores. As an example, oxygen was used to fill the head space of the vial before 

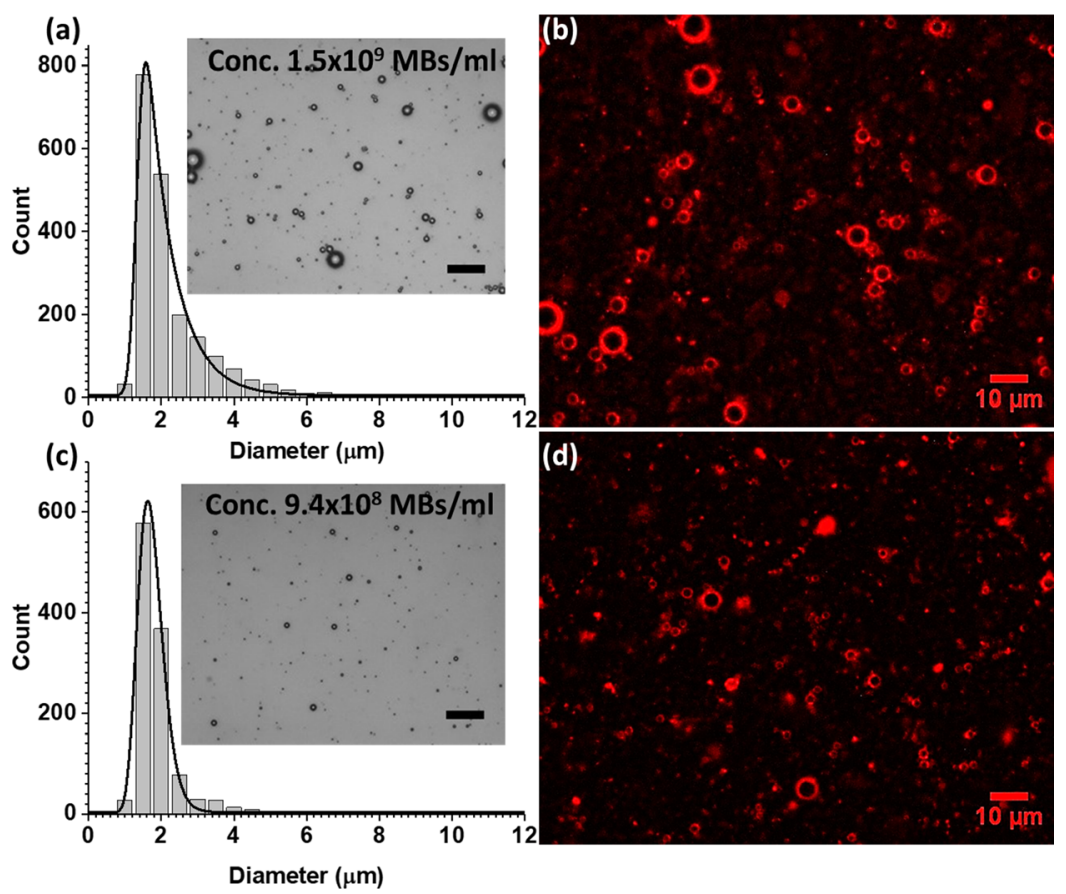

Figure 1. Concentration and mean size of gem.-loaded thMBs before and after FD. (a and c) Histograms showing size distribution of gem.-loaded thMBs (a) before with an average diameter of $2.1 \pm 1.4 \mu \mathrm{m}$ and a concentration of $1.5 \times 10^{9}$ and (c) after with an average diameter of $1.7 \pm 0.7 \mu \mathrm{m}$ and a concentration of $9.4 \times 10^{8}$. The bright field microscopy inset shows MBs before and after FD (scale bar is $10 \mu \mathrm{m}$ ). (b and d) Fluorescence image of thMBs with Texas Red fluorescently tagged gem. liposomes surrounding the thMBs before and after FD, respectively.

adding the hydrating solution and reconstituting the $\mathrm{MBs} . \mathrm{O}_{2}$ release from reconstituted $\mathrm{MBs}$ was tested using an $\mathrm{O}_{2}$ dipping probe (PreSens Precision Sensing GmbH, Regensburg, Germany) and compared to the release profile from freshly prepared $\mathrm{O}_{2} \mathrm{MBs}$. For $\mathrm{O}_{2}$ reconstitution, the lipid shell consisted of DSPC and DSPE-PEG 2000 at $95: 5 \mathrm{~mol} \%$ at a final lipid concentration of $5 \mathrm{mg} / \mathrm{mL}$. The oxygen release profile was performed by placing the oxygen probe in $5 \mathrm{~mL}$ of MilliQ water with continuous stirring to attain a baseline for $1 \mathrm{~min}$ before $250 \mu \mathrm{L}$ of $\mathrm{MBs}$ was added.

\section{RESULTS AND DISCUSSION}

MBs were prepared using the $3 \mathrm{D}$ spray-regime MF chip as previously described. ${ }^{6}$ This typically produces MBs with a mean size of $\sim 2 \mu \mathrm{m}$ at concentrations between 1 and $5 \times 10^{9}$ $\mathrm{MB} / \mathrm{mL}$. The addition of a therapeutic payload of liposomes does not adversely affect the concentration or size. Liposomes fluorescently labeled with Texas Red in the shell were loaded with gem. and used to prepare gem. thMBs. The liposomes were characterized using the Nanosight and typically were around $180 \mathrm{~nm}$ in diameter (Figure S1). Figure la,b shows the thMB size distribution, the inset is the bright field optical image, and Figure $1 \mathrm{~b}$ shows the fluorescence image of gem.loaded thMBs. Figure 1c,d shows the thMB size distribution and images after reconstitution of the freeze-dried product. When reconstituting back in $1 \mathrm{~mL}$ of buffer, there is typically a small drop in MB concentration; however, this can be offset by reconstitution into smaller volumes, which allow bubble concentrations to be controlled. There is also a slight reduction in the mean size of the MBs that results from the loss of the larger sized bubbles. However, as shown from the histogram, there is no shift in the whole population. Importantly, liposomes both retain their fluorescence and remain attached to the MBs post-reconstitution, as shown in the fluorescence image (Figure 1d).
3.1. Long-Term Stability of Freeze-Dried MBs. The FD aliquots were stored at $4{ }^{\circ} \mathrm{C}$, and one aliquot was reconstituted every month for 6 months to measure the MB concentration and size distribution by optical microscopy (Figure 2).

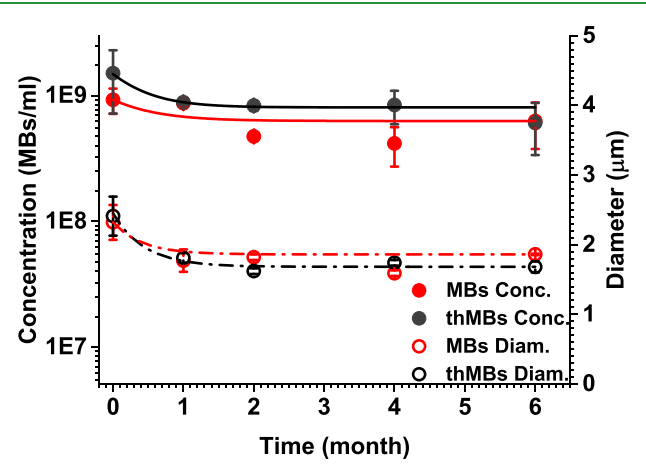

Figure 2. Long-term stability of MBs and liposome-loaded thMBs. $\mathrm{MB}$ concentration and mean diameter are displayed as a function of storage time (in the freeze-dried state). Error bars represent the standard deviation for at least three independent batches of MBs.

There was a slight decrease in average concentration postFD for MBs and thMBs $\left((1.5 \pm 0.7) \times 10^{9}\right.$ to $(9 \pm 0.4) \times 10^{8}$ $\mathrm{MB} / \mathrm{mL}$ ) and also a small decrease in the mean diameter of MBs and thMBs $(2.3 \pm 0.3$ to $1.7 \pm 0.1 \mu \mathrm{m})$. However, these changes were not time-dependent over the period investigated, suggesting that $\mathrm{FD}$ products were stable for at least 6 months when stored at $4{ }^{\circ} \mathrm{C}$. We note that there was no noticeable difference between the $\mathrm{MB}$ and the thMB samples.

Post-reconstitution, the stability of the $\mathrm{MB}$ and thMB samples was tested for up to $4 \mathrm{~h}$ in a closed vial at room temperature and atmospheric pressure. Figure $3 \mathrm{a}, \mathrm{b}$ shows both $\mathrm{MBs}$ and thMBs showing no appreciable change in 

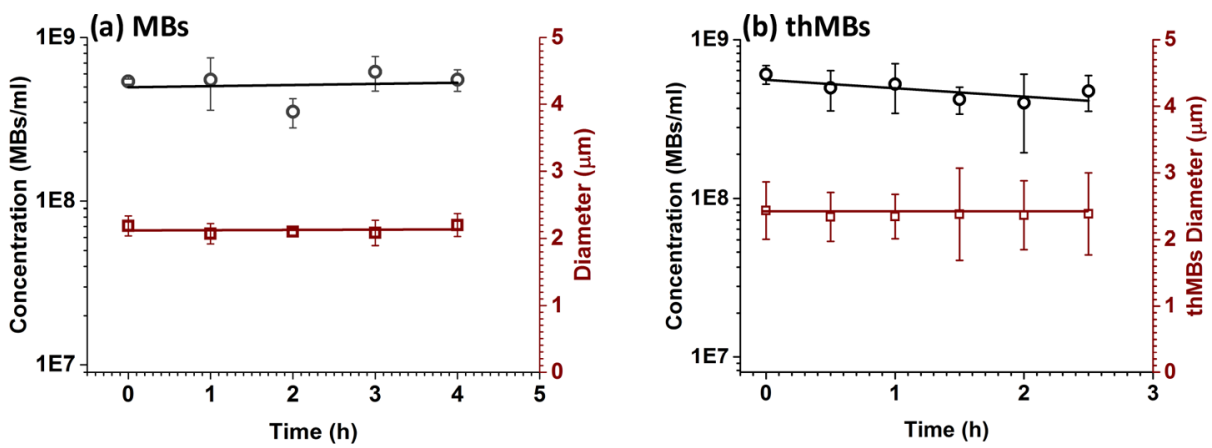

Figure 3. Stability of reconstituted (a) MBs and (b) thMBs. Concentration and size of the MB and thMB populations over $4 \mathrm{~h}$ at room temperature. Open circles represent the concentration, and open squares represent the mean diameter.

concentration or size, providing ample time for MBs to be used post-reconstitution.

3.2. Drug Leakage Test. As a test of liposomal integrity, calcein was loaded into liposomes at $0.5 \mathrm{mM}$, i.e., below the self-quenching concentration (Figure S2). $\mathrm{CoCl}_{2}$ quenches calcein fluorescence, and $100 \mu \mathrm{L}$ of $1 \mathrm{mM} \mathrm{CoCl}_{2}$ was added to the thMB solution post-formation to quench any unencapsulated or free calcein (Figure S3). The graph in Figure 4 shows that with thMBs formed via the conjugation of clean

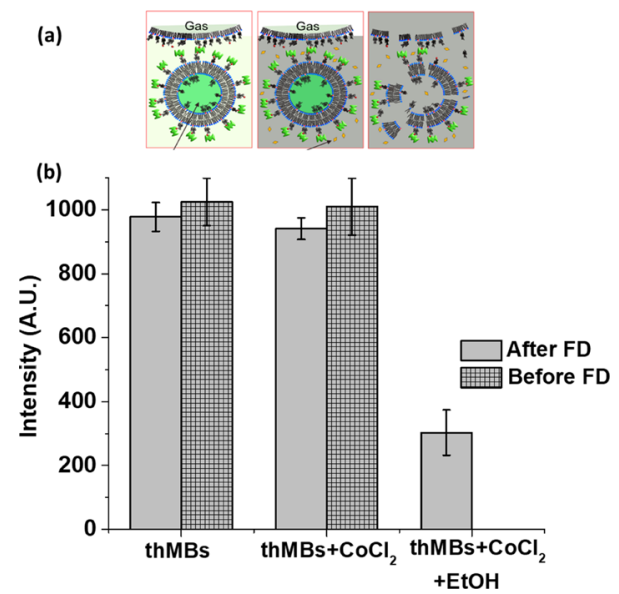

Figure 4. Structural resilience of calcein-filled liposome-loaded MBs to freeze-drying. (a) Schematic showing concept of the leakage assay. (left) Calcein-loaded liposomes were attached to MBs. (middle) $\mathrm{CoCl}_{2}$ was added to the supernatant to quench any fluorescence that leaked from the liposomes. (right) Ethanol was used to break the lipid membrane and release the fluorophore, which was quenched by the surrounding $\mathrm{CoCl}_{2}$. (b) Graph showing the fluorescence from the thMBs post-reconstitution (thMBs), with the addition of $\mathrm{CoCl}_{2}$ $\left(\right.$ thMBs $\left.+\mathrm{CoCl}_{2}\right)$ and following rupture with ethanol (thMBs + $\left.\mathrm{CoCl}_{2}+\mathrm{EtOH}\right)$.

liposomes (cleaned by centrifugation as explained in the Materials and Methods section) to the MB shell, the addition of $\mathrm{CoCl}_{2}$ to such solutions shows only a modest decrease in fluorescence. The addition of ethanol led to the rupture of liposomes and quenching of the fluorescence from the released calcein when in the presence of $\mathrm{CoCl}_{2}$. This demonstrates that not only do the liposomes remain attached to the MBs during the FD process but that they also retain their payload.

The leakage of calcein from liposomes bound to MBs before and after FD is presented in Figure S5, where the fluorescence of the loaded MBs was tested over a $3 \mathrm{~h}$ period at room temperature, either immediately following production or following resuspension post-FD. These results show that post-FD, there is a $\sim 15 \%$ decrease in fluorescence over the subsequent $3 \mathrm{~h}$.

In vivo testing of the US properties of both $\mathrm{MB}$ and gem. thMB populations before and after $\mathrm{FD}$ was performed, and the intensity vs time curves (TICs) were collected from three mice in each case. These curves were assessed for contrast enhancement and stability in the blood stream by imaging the MBs in the aorta and the interior vena cava (highlighted areas) in Figure 5. Echogenicity and stability of the groups
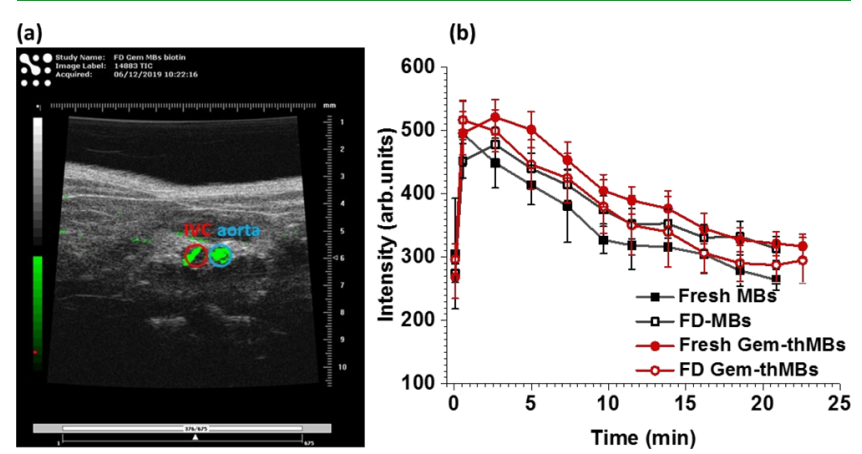

Figure 5. (a) High-frequency contrast-enhanced ultrasound image showing the aorta and the interior vena cava post-injection with gem.thMBs (green-colored signal). (b) Ultrasound time-intensity curves (TICs) in mouse aortas for MBs and gem.-thMBs before and after freeze-drying.

show no statistically significant differences in terms of peak enhancement (contrast enhancement), wash-in slope (rate of MB flow), time to peak enhancement, decay rate (reduction in the contrast enhancement from the peak), area under the curve (indicative of the total area perfused), and full width at halfmaximum (FWHM, the length of time at which the contrast enhancement is $50 \%$ of the peak intensity or greater). Therefore, there are no statistically significant differences in in vivo imaging parameters or lifetime of the MBs.

3.3. Gas Exchange. A potential benefit of freeze-drying $\mathrm{MBs}$ is that it offers a facile way to exchange the composition of the gas core. By simply changing the gas head space during reconstitution, it is possible to formulate MBs with a new gas core. However, we have found that the ability to do this depends on the gas used in the original MB formulation. Figure 6 shows examples of forming $\mathrm{O}_{2}$ MBs post-FD from samples where the original core gas was $\mathrm{O}_{2}$ (Figure $6 \mathrm{a}$ ) or $\mathrm{SF}_{6}$ (Figure $6 \mathrm{c}$ ). When the original core gas was $\mathrm{C}_{4} \mathrm{~F}_{10}$, we were able to reconstitute MBs (Figure $6 \mathrm{~b}$ ); however, in this case, 


\begin{tabular}{|c|c|c|}
\hline & Before FD & After FD \\
\hline $\begin{array}{l}\text { Gas Core } \\
\text { MB size }(\mu \mathrm{m}) \\
\text { MB Conc. }(\mathrm{MBs} / \mathrm{ml}) \\
\text { DSPC+PEG }_{2000}(5 \mathrm{mg} / \mathrm{ml})\end{array}$ & 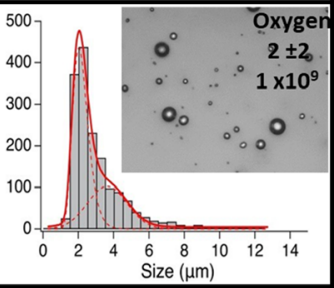 & $\frac{c^{40}}{0}$ \\
\hline 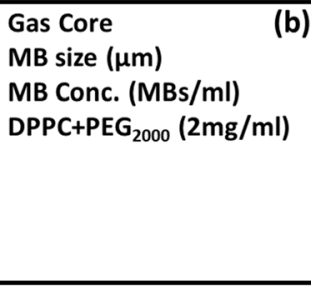 & 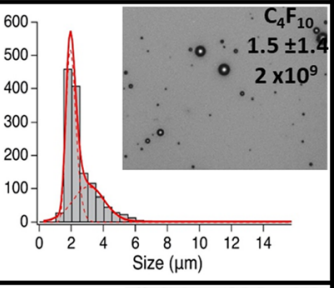 & 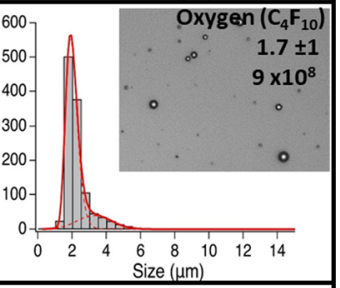 \\
\hline $\begin{array}{l}\text { Gas Core } \\
\text { MB size }(\mu \mathrm{m}) \\
\text { MB Conc. }(\mathrm{MBs} / \mathrm{ml}) \\
\text { DPPC+PEG }_{2000}(2 \mathrm{mg} / \mathrm{ml})\end{array}$ & 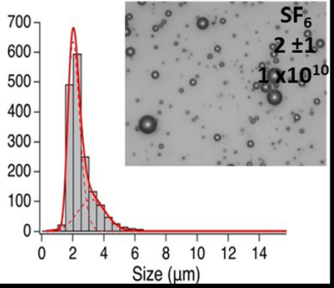 & 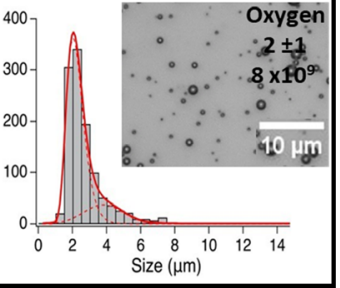 \\
\hline
\end{tabular}

Figure 6. MBs prepared with different gas cores prior to freeze-drying (left) and following rehydration in the presence of oxygen post-freeze-drying (right). Lipid constituents for $\mathrm{MB}$ production alongside $\mathrm{MB}$ concentration and size pre- and post-FD in the presence of $\mathrm{O}_{2}$ are shown. (panel a) MBs prepared with oxygen core in a DSPC-dominated lipid shell and reconstituted after FD in oxygen gas. (panel b) MBs prepared with the $\mathrm{C}_{4} \mathrm{~F}_{10}$ gas core and reconstituted after FD in the presence of oxygen gas. (panel c) $\mathrm{MBs}$ prepared with $\mathrm{SF}_{6}$ and reconstituted after FD in the presence of oxygen gas.
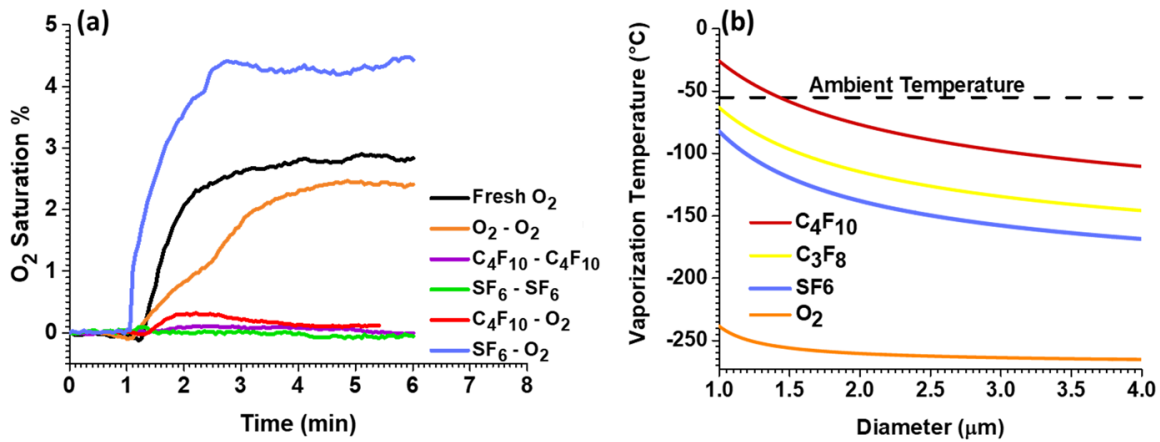

Figure 7. (a) $\mathrm{O}_{2}$ release over time from $\mathrm{MBs}$ prepared with $\mathrm{O}_{2}, \mathrm{C}_{4} \mathrm{~F}_{10}, \mathrm{SF}_{6}$ gas cores prior to freeze-drying and then reconstituted with different gases post-freeze-drying in $5 \mathrm{~mL}$ of water. (b) Predicted vaporization temperature of MBs with different gas cores at $-55^{\circ} \mathrm{C}$ and at $9 \times 10^{-3} \mathrm{mBar}$, representing conditions during the freeze-drying process.

our data (discussed later) suggest that the core was not exchanged and that the gas core remained predominantly $\mathrm{C}_{4} \mathrm{~F}_{10}$ post-reconstitution.

We note that $\mathrm{MBs}$ that had an original $\mathrm{O}_{2}$ core and were then reconstituted in $\mathrm{O}_{2}$ were not stable after $\mathrm{FD}$ and the concentration fell by a factor of two. To compensate for this, the freeze-dried $\mathrm{O}_{2} \mathrm{MBs}$ were usually re-hydrated in half the original volume of solution. This was not seen for the other MBs. It was noticed that oxygen-filled bubbles behave differently from fluorocarbon bubbles, and in the case of post-freeze-drying (Figure 6a), the bubble distribution consists of two populations, the original sized MB plus some corresponding to a larger, possibly merged MBs. This leads to an apparent increase in average size to $\sim 3 \mu \mathrm{m}$. In some cases, these also formed elliptical bubbles. However, this is not the case for $\mathrm{C}_{4} \mathrm{~F}_{10}$ and $\mathrm{SF}_{6}$ post-freeze-drying (Figure $6 \mathrm{~b}, \mathrm{c}$ ) where the size distribution did not show an observable difference after FD. Figure 7 shows the increase in $\mathrm{O}_{2}$ concentration in water following the addition of the different $\mathrm{O}_{2} \mathrm{MBs}$ to MilliQ water. For controls, we also reconstituted $\mathrm{SF}_{6}$ and $\mathrm{C}_{4} \mathrm{~F}_{10}$ back to their original gas core. Upon addition of these control $\mathrm{MBs}$ to a $5 \mathrm{~mL}$ water solution, there was no notable change in $\mathrm{O}_{2}$ levels in the water.

However, $\mathrm{MBs}$ with original gas cores of $\mathrm{O}_{2}$ and $\mathrm{SF}_{6}$ could be reconstituted with $\mathrm{O}_{2}$ post-freeze-drying, and this led to a significant release of $\mathrm{O}_{2}$ into the aqueous solution with $\sim 3 \%$ increase in $\mathrm{O}_{2}$ water saturation level. For the $\mathrm{MBs}$ with a $\mathrm{C}_{4} \mathrm{~F}_{10}$ core but reconstituted with $\mathrm{O}_{2}$, we saw only a small increase in oxygen saturation level post-reconstitution $(<0.5 \%)$, indicating that most of the MBs did not contain significant $\mathrm{O}_{2}$.

During the freeze-drying process, the bubbles are maintained at $-55^{\circ} \mathrm{C}$ under vacuum with an equivalent pressure of $9 \times$ 
$10^{-3}$ mBar. Using a model that we have described previously, ${ }^{54,55}$ we predicted the vaporization temperature of MBs of various sizes with commonly used gas cores under these conditions. With a surface tension of $20 \mathrm{mN} / \mathrm{m}^{6}{ }^{6} \mathrm{SF}_{6}$, $\mathrm{C}_{3} \mathrm{~F}_{8}$, and $\mathrm{O}_{2} \mathrm{MBs}>1 \mu \mathrm{m}$ in diameter would all remain gaseous during freeze-drying. On the contrary, $\mathrm{C}_{4} \mathrm{~F}_{10} \mathrm{MBs}$ with diameters of $\lesssim 1.4 \mu \mathrm{m}$ would remain in a condensed, liquid phase, close to the modal diameter of our MBs. These results suggest that $\mathrm{C}_{4} \mathrm{~F}_{10}$ largely remains in the $\mathrm{FD} \mathrm{MB}$ "cake" such that upon reconstitution, they return to being $\mathrm{C}_{4} \mathrm{~F}_{10} \mathrm{MBs}$ with little $\mathrm{O}_{2}$ content. However, for $\mathrm{C}_{3} \mathrm{~F}_{8}$ and $\mathrm{SF}_{6} \mathrm{MBs}$, the gas core can easily be replaced with $\mathrm{O}_{2}$ after the freeze-drying process.

For all cases, a fixed volume of $250 \mu \mathrm{L}$ of the MB solution was added to $5 \mathrm{~mL}$ of water; however, the $\mathrm{MB}$ concentration was higher in the case of FD SF 6 and is in the order of FD SF 6 $>$ fresh $\mathrm{O}_{2}>\mathrm{FD} \mathrm{O}_{2} \mathrm{MBs}$, which was calculated to be averages of $3.2 \times 10^{8}, 1.4 \times 10^{8}$, and $6 \times 10^{7} \mathrm{MBs}$ in $250 \mu \mathrm{L}$ for each case, respectively. This difference in $\mathrm{MB}$ concentrations is clearly translated to the detected increase in water oxygen concentration in Figure 7.

\section{CONCLUSIONS}

The widespread use of MBs as contrast-enhanced ultrasound agents can be attributed to the ease with which samples can be prepared, from a spray- or freeze-dried product and reconstituted as required. However, this is not the case for small-molecule drug-loaded thMBs. ${ }^{42,47,49,50}$ Here, we have demonstrated that drug-loaded liposomes attached to MBs (thMBs) can be freeze-dried and reconstituted without adversely affecting either the attachment of the liposomes to the MBs or causing leakage of the therapeutic agents from the liposome. Fluorescence microscopy studies showed that the thMBs were still coated with their liposomal payload, and fluorescence leakage assays of a model drug showed that these liposomes remained intact following reconstitution. The freeze-dried thMB product is stable and can be reformulated for time periods of at least 6 months. Furthermore, the stability and echogenic properties of the drug-loaded MBs were maintained in vivo after freeze-drying. Our approach allows large-scale production and storage of thMBs, requiring only a simple reconstitution step prior to use. Once reconstituted, the thMBs have an on-bench lifetime of $>4 \mathrm{~h}$, giving ample time for use. During the reconstitution, we found that as long as the original thMB preparation was made using $\mathrm{SF}_{6}$, then it is possible to exchange the gas core of the thMBs, e.g., to oxygen, which allows improved therapeutic benefit for the treatment of certain diseases.

\section{ASSOCIATED CONTENT}

\section{SI Supporting Information}

The Supporting Information is available free of charge at https://pubs.acs.org/doi/10.1021/acsabm.0c00982.

Liposome size and concentration measured by particle tracking (Nanosight), fluorometer calibration curve for calcein and $\mathrm{CoCl}_{2}$ quenching, fluorometer proof of principle for the $\mathrm{CoCl}_{2}$ quenching effect of calcein, and fluorometer monitoring of calcein leakage from thMBs before and after freeze-drying (PDF)

\section{AUTHOR INFORMATION}

\section{Corresponding Author}

Stephen D. Evans - Molecular and Nanoscale Physics Group, School of Physics and Astronomy, University of Leeds, Leeds LS2 9JT, U.K.; 10 orcid.org/0000-0001-8342-5335; Phone: (+44) (0)113 343 3852; Email: s.d.evans@ leeds.ac.uk

\section{Authors}

Radwa H. Abou-Saleh - Molecular and Nanoscale Physics Group, School of Physics and Astronomy, University of Leeds, Leeds LS2 9JT, U.K.; Biophysics Group, Department of Physics, Faculty of Science, Mansoura University, Mansoura 35511, Egypt; iorcid.org/0000-0002-8471-2659

Aileen Delaney - Molecular and Nanoscale Physics Group, School of Physics and Astronomy, University of Leeds, Leeds LS2 9JT, U.K.

Nicola Ingram - Leeds Institute of Medical Research, St. James's University Hospital, Leeds LS9 7TF, U.K.

Damien V. B. Batchelor - Molecular and Nanoscale Physics Group, School of Physics and Astronomy, University of Leeds, Leeds LS2 9JT, U.K.; 이이.org/0000-0001-6489-9578

Benjamin R. G. Johnson - Molecular and Nanoscale Physics Group, School of Physics and Astronomy, University of Leeds, Leeds LS2 9JT, U.K

Antonia Charalambous - Leeds Institute of Medical Research, St. James's University Hospital, Leeds LS9 7TF, U.K.

Richard J. Bushby - Molecular and Nanoscale Physics Group, School of Physics and Astronomy and School of Chemistry, University of Leeds, Leeds LS2 9JT, U.K.; @ orcid.org/00000002-1627-6058

Sally A. Peyman - Molecular and Nanoscale Physics Group, School of Physics and Astronomy, University of Leeds, Leeds LS2 9JT, U.K.; Leeds Institute of Medical Research, St. James's University Hospital, Leeds LS9 7TF, U.K.; 이이. ord.org/00000002-1600-5100

P. Louise Coletta - Leeds Institute of Medical Research, St. James's University Hospital, Leeds LS9 7TF, U.K.

Alexander F. Markham - Leeds Institute of Medical Research, St. James's University Hospital, Leeds LS9 7TF, U.K.

Complete contact information is available at:

https://pubs.acs.org/10.1021/acsabm.0c00982

\section{Notes}

The authors declare no competing financial interest.

The raw data required to reproduce these findings are available to download from "https://doi.org/10.5518/788".

\section{ACKNOWLEDGMENTS}

We thank the Leeds microbubble consortium for the fruitful discussion and the following funders: MRC (MR/M009084/ 1) and EPSRC (EP/P023266/1). S.D.E. is supported by the National Institute for Health Research (NIHR) infrastructure at Leeds. The views expressed are those of the author(s) and not necessarily those of the NHS, the NIHR, or the Department of Health.

\section{REFERENCES}

(1) Klibanov, A. L. Microbubble contrast agents: targeted ultrasound imaging and ultrasound-assisted drug-delivery applications. Invest. Radiol. 2006, 41, 354-362.

(2) Lindner, J. R. Microbubbles in medical imaging: current applications and future directions. Nat. Rev. 2004, 3, 527-533. 
(3) Lindner, J. R.; Ismail, S.; Spotnitz, W. D.; Skyba, D. M.; Jayaweera, A. R.; Kaul, S. Albumin microbubble persistence during myocardial contrast echocardiography is associated with microvascular endothelial glycocalyx damage. Circulation 1998, 98, 21872194.

(4) Stride, E.; Saffari, N. Microbubble ultrasound contrast agents: a review. Proc. Inst. Mech. Eng. Part H 2003, 217, 429-447.

(5) Peyman, S. A.; Abou-Saleh, R. H.; Evans, S. D. Research Spotlight: Microbubbles for therapeutic delivery. Ther. Delivery 2013, 4, 539-542.

(6) Abou-Saleh, R. H.; Peyman, S. A.; Johnson, B. R. G.; Marston, G.; Ingram, N.; Bushby, R.; Coletta, P. L.; Markham, A. F.; Evans, S. D. The influence of intercalating perfluorohexane into lipid shells on nano and microbubble stability. Soft Matter 2016, 12, 7223-7230.

(7) Klibanov, A. L.; Hughes, M. S.; Wojdyla, J. K.; Wible, J. H., Jr.; Brandenburger, G. H. Destruction of contrast agent microbubbles in the ultrasound field: the fate of the microbubble shell and the importance of the bubble gas content. Acad Radiol. 2002, 9, S41-S45.

(8) Kukizaki, M.; Baba, Y. Effect of surfactant type on microbubble formation behavior using Shirasu porous glass (SPG) membranes. Colloid Surf. A 2008, 326, 129-137.

(9) Kwan, J. J.; Borden, M. A. Microbubble dissolution in a multigas environment. Langmuir 2010, 26, 6542-6548.

(10) Kwan, J. J.; Borden, M. A. Lipid monolayer collapse and microbubble stability. Adv. Colloid Interface Sci. 2012, 183-184, 8299.

(11) Kooiman, K.; Vos, H. J.; Versluis, M.; de Jong, N. Acoustic behavior of microbubbles and implications for drug delivery. Adv. Drug Deliver Rev. 2014, 72, 28-48.

(12) Mclaughlan, J. R.; Harput, S.; Abou-Saleh, R. H.; Peyman, S. A.; Evans, S.; Freear, S. Characterisation of Liposome-Loaded Microbubble Populations for Subharmonic Imaging. Ultrasound Med. Biol. 2017, 43, 346-356.

(13) Feinstein, S. B. The powerful microbubble: from bench to bedside, from intravascular indicator to therapeutic delivery system, and beyond. Am. J. Physiol. Heart Circ. Physiol. 2004, 287, H450$\mathrm{H} 457$.

(14) Suzuki, R.; Oda, Y.; Omata, D.; Nishiie, N.; Koshima, R.; Shiono, Y.; Sawaguchi, Y.; Unga, J.; Naoi, T.; Negishi, Y.; Kawakami, S.; Hashida, M.; Maruyama, K. Tumor growth suppression by the combination of nanobubbles and ultrasound. Cancer Sci. 2016, 107, 217-223.

(15) McLaughlan, J. R.; Ingram, N.; Abou-Saleh, R. H.; Harput, S.; Evans, T.; Evans, S. D.; Coletta, L.; Freear, S. High-frequency subharmonic imaging of liposomes loaded microbubbles. In 2013 IEEE International Ultrasonics Symposium (IUS); IEEE: Prague, 2125 July 2013; Prague, 2013; pp. 1501-1504.

(16) Unger, E. C.; Porter, T.; Culp, W.; LaBell, R.; Matsunaga, T.; Zutshi, R. Therapeutic applications of lipid-coated microbubbles. Adv. Drug. Deliver Rev. 2004, 56, 1291-1314.

(17) Mico, V.; Charalambous, A.; Peyman, S. A.; Abou-Saleh, R. H.; Markham, A. F.; Coletta, P. L.; Evans, S. D. Evaluation of lipidstabilised tripropionin nanodroplets as a delivery route for combretastatin A4. Int. J. Pharm. 2017, 526, 547-555.

(18) Lentacker, I.; De Smedt, S. C.; Sanders, N. N. Drug loaded microbubble design for ultrasound triggered delivery. Soft Matter 2009, 5, 2161-2170.

(19) Lentacker, I.; Geers, B.; Demeester, J.; De Smedt, S. C.; Sanders, N. N. Tumor cell killing efficiency of doxorubicin loaded microbubbles after ultrasound exposure. J. Controlled Release 2010, 148, e113-e114.

(20) Geers, B.; Lentacker, I.; Sanders, N. N.; Demeester, J.; Meairs, S.; De Smedt, S. C. Self-assembled liposome-loaded microbubbles: The missing link for safe and efficient ultrasound triggered drugdelivery. J. Controlled Release 2011, 152, 249-256.

(21) Segers, T.; Lassus, A.; Bussat, P.; Gaud, E.; Frinking, P. Improved coalescence stability of monodisperse phospholipid-coated microbubbles formed by flow-focusing at elevated temperatures. Lab Chip 2019, 19, 158-167.
(22) Shih, R.; Lee, A. P. Post-Formation Shrinkage and Stabilization of Microfluidic Bubbles in Lipid Solution. Langmuir 2016, 32, 19391946.

(23) Xu, Q.; Nakajima, M.; Ichikawa, S.; Nakamura, N.; Shiina, T. A comparative study of microbubble generation by mechanical agitationand sonication. Innovative Food Sci. Emerging Technol. 2008, 9, 489-494.

(24) Qin, S.; Caskey, C. F.; Ferrara, K. W. Ultrasound contrast microbubbles in imaging and therapy: physical principles and engineering. Phys. Med. Biol. 2009, 54, R27-R57.

(25) Hashimoto, M.; Whitesides, G. M. Formation of bubbles in a multisection flow-focusing junction. Small 2010, 6, 1051-1059.

(26) Peyman, S. A.; Abou-Saleh, R. H.; McLaughlan, J. R.; Ingram, N.; Johnson, B. R. G.; Critchley, K.; Freear, S.; Evans, J. A.; Markham, A. F.; Coletta, P. L.; Evans, S. D. Expanding 3D geometry for enhanced on-chip microbubble production and single step formation of liposome modified microbubbles. Lab Chip 2012, 12, 4544-4552.

(27) Talu, E.; Hettiarachchi, K.; Powell, R. L.; Lee, A. P.; Dayton, P. A.; Longo, M. L. Maintaining monodispersity in a microbubble population formed by flow-focusing. Langmuir 2008, 24, 1745-1749.

(28) Segers, T.; Gaud, E.; Casqueiro, G.; Lassus, A.; Versluis, M.; Frinking, P. Foam-free monodisperse lipid-coated ultrasound contrast agent synthesis by flow-focusing through multi-gas-component microbubble stabilization. Appl. Phys. Lett. 2020, 116, 173701.

(29) Peyman, S. A.; Abou-Saleh, R. H.; Evans, S. D. Bubble generation. 2013001309, 2013.

(30) Sarkar, K.; Katiyar, A.; Jain, P. Growth and dissolution of an encapsulated contrast microbubble: effects of encapsulation permeability. Ultrasound Med Biol 2009, 35, 1385-1396.

(31) Schutt, E. G.; Evitts, D. P.; Anderson, C. D.; Weers, J. G. Mixed gas microbubble composition. US 6,953,569 B2, 2005.

(32) Kabalnov, A.; Bradley, J.; Flaim, S.; Klein, D.; Pelura, T.; Peters, B.; Otto, S.; Reynolds, J.; Schutt, E.; Weers, J. Dissolution of multicomponent microbubbles in the bloodstream: 2 Experiment. Ultrasound Med. Biol. 1998, 24, 751-760.

(33) Kabalnov, A. S.; Makarov, K. N.; Scherbakova, O. V. Solubility of fluorocarbons in water as a key parameter determining fluorocarbon emulsion stability. J. Fluorine Chem. 1990, 50, 271-284.

(34) Hua, Z. Z.; Li, B. G.; Liu, Z. J.; Sun, D. W. Freeze-Drying of Liposomes with Cryoprotectants and Its Effect on Retention Rate of Encapsulated Ftorafur and Vitamin A. Drying Technol. 2003, 21, $1491-1505$.

(35) Jennings, T. A. Lyophilization: Introduction and basic principles; 1st edition ed. Taylor and Francis group: 1999; p 664.

(36) Franks, F. Freeze-drying of bioproducts: putting principles into practice. Eur.J.Pharm. Biopharm 1998, 45, 221-229.

(37) Tang, X. C.; Pikal, M. J. Design of freeze-drying processes for parmaceuticals: Practical advice. Pharm. Res. 2004, 21, 191-200.

(38) Abdelwaheb, W.; Degobert, G.; Stainmesse, S.; Fessi, H. Freeze-drying of nanoparticles: formulation, process and storagr considerations. Adv. Drug Delivery Rev. 2006, 58, 1688-1713.

(39) Song, S. W.; Bae, H. J.; Kim, S.; Oh, D. Y.; Kim, O.; Jeong, Y.; Kwan, S. Uniform Drug Loading into Prefabricated Microparticles by Freeze-Drying. Part. Part. Syst. Charact. 2017, 34, 1-7.

(40) Christensen, D.; Kirby, D.; Foged, C.; Agger, E. M.; Andersen, P.; Perrie, Y.; Nielsen, H. M. $\alpha, \alpha^{\prime}$-trehalose 6,6'-dibehenate in nonphospholipid-based liposomes enables direct interaction with trehalose, offering stability during freeze-drying. Biochim. Biophys. Acta 2008, 1778, 1365-1373.

(41) van Winden, E. C. A. Freeze-drying of liposomes: theory and practice. liposomes, Pt, A 2003, 367, 99-110.

(42) Solis, C.; Forsberg, F.; Wheatley, M. A. Preserving enhancement in freeze-dried contrast agent ST68: examination of excipients. Int. J. Pharm. 2010, 396, 30-38.

(43) Schneider, M.; Brochot, J.; Puginier, J.; Yan, F. Stable microbubble suspensions comprising saturated. 5,686,060, 1997.

(44) Sontum, P. C. Physicochemical characteristics of Sonazoid (TM), a new contrast agent for ultrasound imaging. Ultrasound Med. Biol. 2008, 34, 824-833. 
(45) Sontum, P. C.; Østensen, J.; Dyrstad, K.; Hoff, L. Acoustic properties of NC100100 and their relation with the microbubble size distribution. Invest. Radiol. 1999, 34, 268-275.

(46) Borden, M. A.; Swanson, E. J. Systems, methods, and devices for microbubbles. 9,107,950, 2015.

(47) Eisenbrey, J. R.; Albala, L.; Kramer, M. R.; Daroshefski, N.; Brown, D.; Liu, J.-B.; Stanczak, M.; O’Kane, P.; Forsberg, F.; Wheatley, M. A. Development of an ultrasound sensitive oxygen carrier for oxygen delivery to hypoxic tissue. Int. J. Pharm. 2015, 478, $361-367$.

(48) Ojha, T.; Pathak, V.; Drude, N.; Weiler, M.; Rommel, D.; Rutten, S.; Geinitz, B.; van Steenbergen, M. J.; Storm, G.; Kiessling, F.; Lammers, T. Shelf-Life Evaluation and Lyophilization of PBCABased Polymeric Microbubbles. Pharmaceutics 2019, 11, 1-13.

(49) Unga, J.; Omata, D.; Kudo, N.; Ueno, S.; Munakata, L.; Shima, T.; Suzuki, R.; Maruyama, K. Developement and evaluation of stability and ultrasound response of DSPC-DPSG-based freeze-dried microbubbles. J Liposome Res 2019, 4, 368-374.

(50) Unga, J.; Kageyama, S.; Suzuki, R.; Omata, D.; Maruyama, K. Scale-up production, characterization and toxicity of a freeze-dried lipid-stabilized microbubble formulation for ultrasound imaging and therapy. J. Liposome Res. 2019, 1-304.

(51) Shafi, A. S.; McClements, D. J.; Al-Baijan, I.; Abou-Saleh, R. H.; Moran, C.; Koutsos, V. Probing Phospholipid-Microbubbles by Atomic Force Microscopy to Quantify Bubble Mechanics and Nanostructural Shell Properties. Colloids Surf., B 2019, 181, 506-515.

(52) Batchelor, D. DamienBatchelor/UoL_MicrobubbleCount: First public release of MATLAB Microbubble Code (Version v1.0.1). Zenodo. 20/07/2020 ed.; 2020.

(53) Zhai, L.; Zhao, J.; Zhao, M.; Chen, Y.; Zhang, L. Encapsulation and releasing of calcein by spontaneously formed zwitterionic/anionic vesicle without separation. J. Dispersion Sci. Technol. 2007, 28, 445461.

(54) Batchelor, D. V. B.; Abou-Saleh, R. H.; Coletta, P. L.; McLaughlan, J. R.; Peyman, S. A.; Evans, S. D. Nested Nanobubbles for Ultrasound-Triggered Drug Release. ACS Appl. Mater. Interfaces 2020, 12, 29085-29093.

(55) Doinikov, A. A.; Sheeran, P. S.; Bouakaz, A.; Dayton, P. A. Vaporization dynamics of volatile perfluorocarbon droplets: a theoretical model and in vitro validation. Med Phys 2014, 41, 102901. 